\title{
Effective Teleconsulting and Teleconferencing Using Standard DICOM Protocol and Push Technology on Low Bandwidth Connection
}

\author{
P Marcheschi, A Mazzarisi, P Marraccini, M Glauber, D Chiappino, A Benassi \\ CNR Institute of Clinical Physiology, Pisa, Italia
}

\begin{abstract}
Teleconsulting and Teleconferencing is nowadays a strict need for patient follow-up or for medical second opinion. The growing of this kind of techniques for teleconsulting needs an effective way to achieve a fast communication on non-dedicated connection lines. Moreover another prerequisite for this kind of activities is the conformance to DICOM that is the de facto standard for medical image storage and communication. The present work proposes a way to distribute medical images by means of standard telecommunications media (i.e. Ethernet lines, Modem Lines, ISDN Lines), and in standard DICOM format using freely available opensource tools.
\end{abstract}

\section{Introduction}

Teleconsulting and teleconferencing are nowadays an irreplaceable means for the medical profession, unfortunately the solutions proposed by the market are inefficient or enormously expensive. However, there are a lot of possibilities offered by the modern computer technology. In fact every personal computer is equipped with teleconferencing tools and suites of protocols that permit information sharing through the world wide web. There is, besides, the necessity to stick to medical standard of primary importance, which is for instance the Digital Imaging and Communication protocol (DICOM)[10]. The purpose of this work, is to fully exploit the possibilities offered by the modern technology, sticking to the standards, creating so a background for further developments in teleconsulting. The realized system is made up by: a dedicated Linux based communication broker server that is able to understand DICOM way of communication, is able to compress images using standard jpeg lossy or lossless method, and is able to redistribute them to other DICOM nodes over the network.

The broker server can receive automatically images from an acquisition modality such as a Magnetic Resonance Imaging (MRI), a Computer Tomography (CT), a Digital Angiography (XA) or other modalities, acting as a DICOM SCP (Service Class Provider) for the storage service.

It can compress each frame of every sequence in a chosen compressed DICOM standard format. It can, optionally, anonymize, redistribute images and sequences to the selected nodes over the network in standard way for local area network communication or with DICOM TLS (Transport Layer security) support to crypt sensible data over insecure networks (Internet), acting as a SCU (Service Class User) for the storage DICOM service.

The problems connected to the way of making available the images and data, through a network are manifold:

1. it is necessary to distribute the data in a simple way, or rather in such a way that doesn't impact the life of the technical personnel turned to the treatment of such information;

2. it is necessary to respect all those security criteria for reservation of the data imposed by the law;

3. it is necessary to respect the normal activities developed inside the medical environment.

To fulfil these tasks we choose to stick completely to the DICOM standard, and to adapt our applications to it through a DICOM broker.

\section{System overview}

The fulcrum of the system is constituted by a computer that acts as broker for the data that are treating.

Such computer is based on Linux on $\mathrm{x} 86$ platform, the choice of such platform is mainly dictated by low cost, convenience of use and stability. Besides, numerous open-source products for the development of medical network applications are present on Linux. To create the demon that performs the work in object, we have used the Perl programming language. For the part of transmission and conversion in standard DICOM, we have used the DCMTK Offis suite, and for the part of anonymization and correction of unwanted fields in the data, the suite D. Clunie dicomtools [11]. Every diagnostic modality that understands, in its own software endowment, the DICOM standard, allows sending toward a DICOM Server a selection of images selected by the operator.

Therefore it is possible to exploit this possibility to transfer the required material for the consultation in such a way that the diagnostic modality sees a normal DICOM server on the net.

As regards, the receiving application, it behaves as a service class provider, in order to accept images from a 
service class user for the service of storage.

Besides, such application when realizes that the transfer of a complete image study has already taken place, acts consequently: at the reception of the images the program extracts the necessary information to start the transfer to the multiple teleconsulting nodes. In fact it is possible to program the DICOM broker to send the study to multiple receiving workstations. The only prerequisite is the DICOM compliance. The application that attends the broker service, on receiving of the DICOM files from the diagnostic modality, completes the followings footsteps:

1. It inspects the transferred files;

2. It recognizes the diagnostic modality

3. It imposes the AE-titles and the network address of destination workstations;

4. It checks if such workstations have been turned on and that the servers have been activated;

5. It optionally completes the anonymization of the files;

6. It eventually straps bugs or inaccuracies of the DICOM format;

7. It unrolls the files;

8. It compresses the images taking advantage of the DICOM jpeg compression variants;

9. It effects the transmission toward the destination workstations;

10. It prepares a report on the effected assignments;

11. It warns through windows messaging protocol the employed operator on the result of the task.

As regards the client part a commercial program has been used. The used program is eFilm, from the Merge eFilm (eFilm Merge, Toronto, Canada). Such program has been selected for the extreme facility of use, practicality and low cost, besides it contains a DICOM server able to accept compressed DICOM streams. This feature permits to receive jpeg compressed DICOM files in order to reduce the occupied bandwidth. Efilm is besides an easy-to-use program for a physician or a radiologist since it contains all the tools present in more powerful visualization workstations, like enhanced 3D/MIP, MPR, and Measurement Tools, it is therefore a good starting point to put into practice a teleconsulting session. It is also possible to implement a full opensource solution, not using eFilm from Merge technologies, but using all opensource software.

For example using Xmedcon for the visualization and the dcmtk dicom server from Offis for the reception of the dicom files. But at the moment the medical staff prefers an integrated commercial solution that is easier to maintain and use. In practice the program completes a series of operations on every single file arrived. The operations of transfer and conversion are monitored and checked by the DICOM Broker, in order to warn the local operator, typically the radiology technician, in the case some operations do not have a successful conclusion, through the dispatch of opportune popup messages. This is made possible by the use of the Microsoft messaging protocol that allows to send messages from a workstation unix (Linux) to a microsoft windows local system via the windows messaging protocol for Samba [14].

\subsection{Network security}

To be able to transmit without any risks of interception the data through the net it is necessary to take some safety measures. The possible scenarios, supported by our application are essentially four:

1. Unencrypted transmission, qualified only for firewall protected local area networks,

2. DICOM TLS (Transfer Layer Security) Transmission, directly supported by the DICOM protocol,

3. Secure transmission through an S-Tunnel connection,

4. Anonymized Data transmission.

These four scenarios can be used in concomitance with techniques of compression of the digital images, to be able to exploit completely the band assigned for the transmission. In fact it is known that the crypt transmission introduces a showy deceleration of the communication not allowing using it in emergency cases. The essential reason for this type of choices is that to cover all the possible situations present in a real world model. Indeed, for instance the TLS, having been implemented also inside the standard DICOM, it is not present unfortunately in none of the commercial visualization workstation implementations we have tried. To be able to utilize the TLS protocol we have used a gateway on the receiving computer able to talk TLS on one side and to internally postpone the images to the DICOM server present on the same computer. Stunnel is a program that allows to encrypt arbitrary TCP connections inside SSL, this solution allows to use a sure abutment in which to make travel information without hitting with the facility of use of the system. The last scenario, the anonymization, is surely to prefer when the network bandwidth is low, i.e. on ISDN or modem connections. TLS (Transport Layer Security) is a protocol born with the purpose to guarantee the privacy of the communications on Internet; it derives from the protocol SSL (Secure Socket Layer), an open protocol whose last version is the 3.0; TLS 1.0 (the actual version) it is very similar to SSL 3.0 nevertheless it is not compatible with this, the implementations however, generally support both TLS 1.0 and SSL 3.0, and the interoperability is guaranteed. The purpose of TLS is that to allow a sure communication among two applications, supplying to the flux of data between them three fundamental functionalities: confidentiality, computer entity authentication, integrity.

Confidentiality, to guarantee a secure connection among two clients involved in a communication, the data is 
protected using algorithms of symmetrical key cryptography; The authentication of the identity in the connections can be performed using the public key cryptography. In such way the consumers are sure to communicate with the correct server preventing possible interpositions; Integrity, the level of transport includes a follow-up on the integrity of the message based on a special message authentication code (MAC) that uses secure hash functions. In such way there is an audit that the transmitted data between client and server have not been altered during the communication.

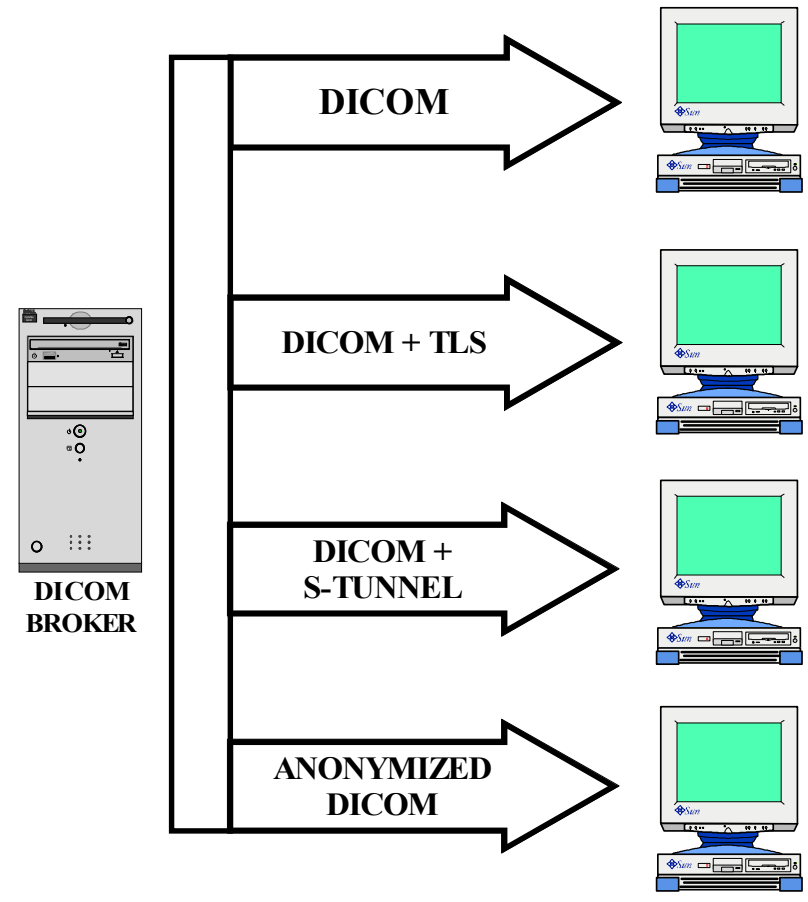

Figure 1. DICOM Security connection scenarios

\subsection{Image compression}

The compression of images is a valid tool in all that cases in which the lossy compression doesn't provoke a damage in the interpretation of the images for diagnostic purpose. Besides it has been shown [5] that compression ratio of $15: 1$ is the visually lossless threshold for the jpeg lossy algorithm. However the acceptable Levels of compression strongly depend on the type of image, infact the technology of the production of medical images has made giant footsteps in the last few years, increasing notably the signal to noise ratio of the created images.

But the possibility to compare directly lossy compressed images with original uncompressed images via the teleconferencing application with the physician who has performed the examination removes every doubt on the correctness of the interpretation.

\subsection{Teleconferencing}

To be able to perform a teleconferencing session in real time, we used a very well known application for the everyday internet user: Microsoft's netmeeting. This application is the microsoft mutimedia conferencing tool present in every standard installation of microsoft windows. It enables audio and video conferencing session, drawing on shared whiteboard, this is very useful for example for cardiac inteventistic procedures. Such application allows to converse through the H263 standard for IP/Ethernet LAN multimedia conferencing using a simple web-cam and a microphone installed on a personal computer. The audio codec uses ITU (International Telecommunication Union) G.723 and offers $4.8 \mathrm{kbps}$ to $64 \mathrm{kbps}$ transfer rate, the video codec uses ITU H263 standards and supports $30 \mathrm{fps}$ frame rate. Besides it is possible to use the whiteboard and the transfer of file for further close examinations. Effected tests show that the quality of the reception is acceptable even on non dedicated lines, like modem connections.

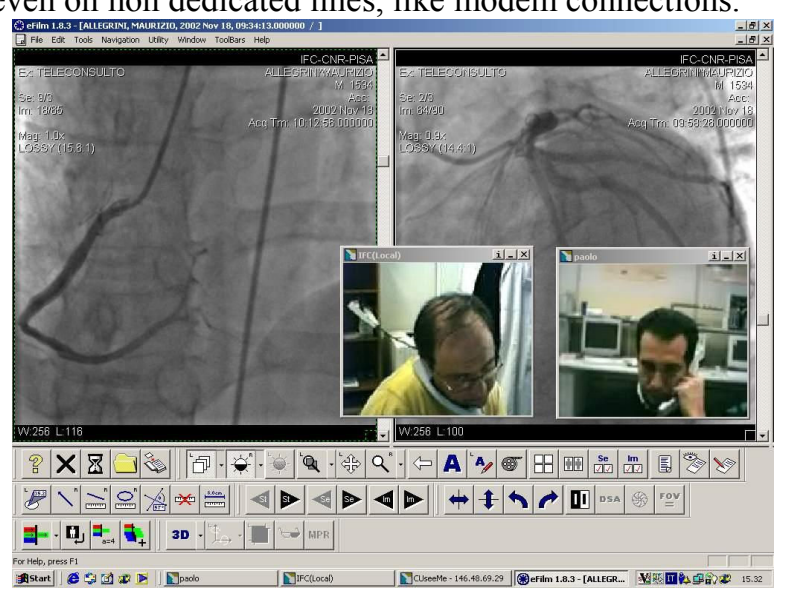

Figure 2. Example of teleconferencing with net meeting and Efilm.

\section{Conclusions}

The here proposed method of teleconsulting and teleconferencing is at the moment in experimentation and evaluation phase. In this period radiology technicians and physicians are getting used to the new way of interacting with radiological information.

We stated that the teleconferencing tool improves a lot the interaction quality, but some physicians still prefer the telephone call to discuss the case. We find that the image compression ratio of 15:1 is suitable for our digital acquisition modalities, but it is too much for analog devices where further noise is introduced in acquisition and in the analog to digital conversion.

Actually more than 30 transfers have taken place using the proposed method, every day the service is sharpened to meet the new claims from the medical staff and is becoming more and more a necessity for medical practice.

\section{Acknowledgements}

The authors would like to thank the radiology technicians of the Institute of Clinical Physiology, and particularly Micaela Papini, Alessandra Perinazzo and 
Giuseppe Maltinti for the support and suggestions in the experimentation still in progress.

\section{References}

[1] Wallace, GK, The JPEG still picture compression standard. Communications of the ACM, 1991, 34, 31, 44.

[2] Rigolin VH, Robiolio PA, Spero LA. Compression of digital coronary angiograms does not affect visual or quantitative assessment of coronary artery stenosis severity. Am J Cardiol 1996 pp. 131-135.

[3] Koning, G, Beretta P, Zwart P, Hekking E, Reiber JH. Effect of lossy data compression on quantitative coronary measurements. Int J Card Imaging 1997;261-270.

[4] American College of Radiology (ACR), Standard for Teleradiology, www.acr.org, 1998.

[5] Kocsis, O, Costaridou L, Mandellos G, Lymberopoulos D, Panayiotakis, G.Compression Assessment based on medical image quality concepts using computer-generated test imagesComputer Methods and Programs in Biomedicine Volume: 71, Issue: 2, June, 2003;105-115.

[6] Kivijarvi J, Ojala T, Kaukoranta T, Kuba A, Nyul L, Nevalainen O. A comparison of lossless compression methods for medical images. Comp. Med. Imag. Graph. 1998;323-339.
[7] Huang HK, "Teleradiology technologies and some service models" Comp. Med. Imag. Graph. 1996;59-68

[8] Video conferencing cookbook: Available at http:/www.videnet.gatech.edu/cookbook/. Accessed Sept 2003.

[9] Xmedcon Medical Image Conversion. Available at http://xmedcon.sourceforge.net/docs/index.html Accessed Sept 2003.

[10] DICOM official website, Available at http://www.nema.org/. Accessed Sept 2003.

[11] David Clunie's Medical Image Format Site, Available at http://www.dclunie.com/. Accessed Sept 2003.

Address for correspondence.

P. Marcheschi

CNR Institute of Clinical Physiology

CNR Research Area - Via G. Moruzzi 1

56124 PISA (ITALY)

E-mail address: paolo@ifc.cnr.it 